\title{
Biologische Rekonstruktionsmöglichkeiten nach Tumorresektion
}

\author{
Ulf Brunnemer, Christian Krettek, Thomas Gösling
}

\section{Zusammenfasssung}

Resektionen muskuloskeletaler Tumoren machen häufig knöcherne Rekonstruktionen der resezierten Knochenstrukturen notwendig. Für die Wiederherstellung des entstandenen ossären Defekts ergeben sich autologe und allogene Lösungen sowie der prothetische Knochenersatz. Bei ausreichender Reststabilität kann gelegentlich auf eine knöcherne Rekonstruktion komplett verzichtet werden und eine temporäre Modifikation der Belastung oder Immobilisation ist ausreichend. Als Autograft findet die Transplantation von Spongiosa, trikortikalen Knochenspänen, gefäßgestielten Knochentransplantaten (Klavikula, Fibula) Anwendung. Als sekundäre Rekonstruktionsmöglichkeit für Röhrenknochen eignet sich die Kallusdistraktion mittels Segmenttransport. Die Verwendung von Allografts ist mit einer hohen Komplikationsrate behaftet und findet kaum Anwendung. Die Umkehrplastik nach Borggreve ist eine alternative Rekonstruktionsmöglichkeit bei Tumoren des Femurs bzw. des proximalen Unterschenkels. Lediglich temporär geschwächte Knochen können mit einer temporären Osteosynthese stabilisiert werden.
Possibilities for Biological Reconstruction after Tumour Resection

The resection of musculoskeletal tumours often makes reconstruction of the resected bone structure necessary. There are both autologous and allogenous options as well as prosthetic bone substitutes for reconstruction of the created bone defect. In cases with sufficient residual stability a boney reconstruction may not be necessary and a temporary modification of loadbearing or immobilisation will give satisfactory results. Spongey bone, tricortical bone chips, or vascular pedicled bone grafts (clavicle, fibula) can be used as autogenous grafts. Callus distraction by means of segment transport is suitable as a secondary reconstruction option for hollow bones. The use of allogenous grafts is accompanied by a high rate of complications and is rarely employed. The reversal plasty according to Borggreve offers a further option for reconstruction in cases of tumours of the femur or, respectively, the proximal lower leg. Bones that are merely temporarily weakened can be stabilised with the help of a temporary osteosynthesis.

\section{Einleitung}

In Abhängigkeit der präoperativ detektierten Tumorentität und der Lokalisierung ist bei muskuloskeletalen Tumoren eine intraläsionale, marginale, weite oder radikale Tumorresektion notwendig. Eine Erweiterung des Resektionsrands kann durch adjuvante lokale Maßnah-

OP-JOURNAL 2010; 26: 196-200

(c) Georg Thieme Verlag KG Stuttgart · New York DOI http://dx.doi.org/10.1055/s-0030-1250618
Prinzipiell kommen biologische und prothetische Lösungen zum Einsatz. Die biologische Knochenrekonstruktion zeichnet sich durch zahlreiche Vorteile aus, wie Langlebigkeit durch Anpassung an Veränderungen der mechanischen Belastung und der Fähigkeit des Längenwachstums beim Kind und Jugendlichen. Die operativen Eingriffe sind häufig technisch anspruchsvoll und bleiben dem Erfahrenen vorbehalten. In der Regel sind strenge Nachbehandlungsregime wie passagere Teilbelastung, temporäre Karenz von Sport, Physiotherapie und eine enge Nachsorge notwendig. Prothetischer Knochenersatz hingegen verfügt über eine hohe Primärstabilität, die operativen Eingriffe sind standardisierter und dank moderner modularer Prothesensysteme gerade beim Erwachsenen individuell anzupassen. Bekannte Nachteile von Prothesen können Implantatversagen wie Lockerungen oder Bruch, allergische Reaktionen oder implantatassoziierte Infekte sein.

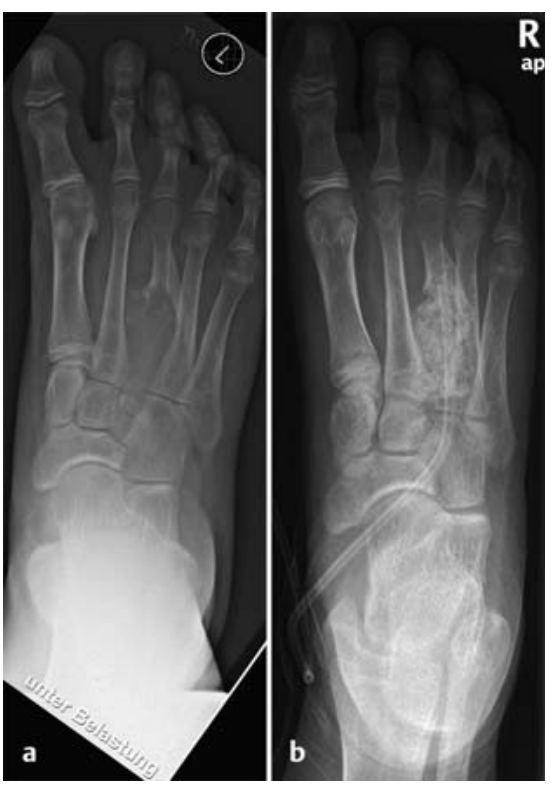

Abb. $1 \mathbf{a}$ und $\mathbf{b}$ a Aneurysmatische Knochenzyste am Os metatarsale III. b Defektauffüllung durch Transplantation von autologer Spongiosa am Os metatarsale III. men wie bspw. die Hitzeentwicklung von passager implantiertem PMMA (,polymethyl methacrylat") erreicht werden. Auch für Ethanol, Phenol und Wasserstoffperoxid sowie die Kryotherapie mit flüssigem Stickstoff existieren entsprechende Empfehlungen in der Literatur. Insbesondere semimaligne oder maligne Tumoren machen in der Regel weite oder radikale Knochenresektionen notwendig, die im Anschluss häufig technisch anspruchsvolle, diffizile Rekonstruktionen erfordern. 


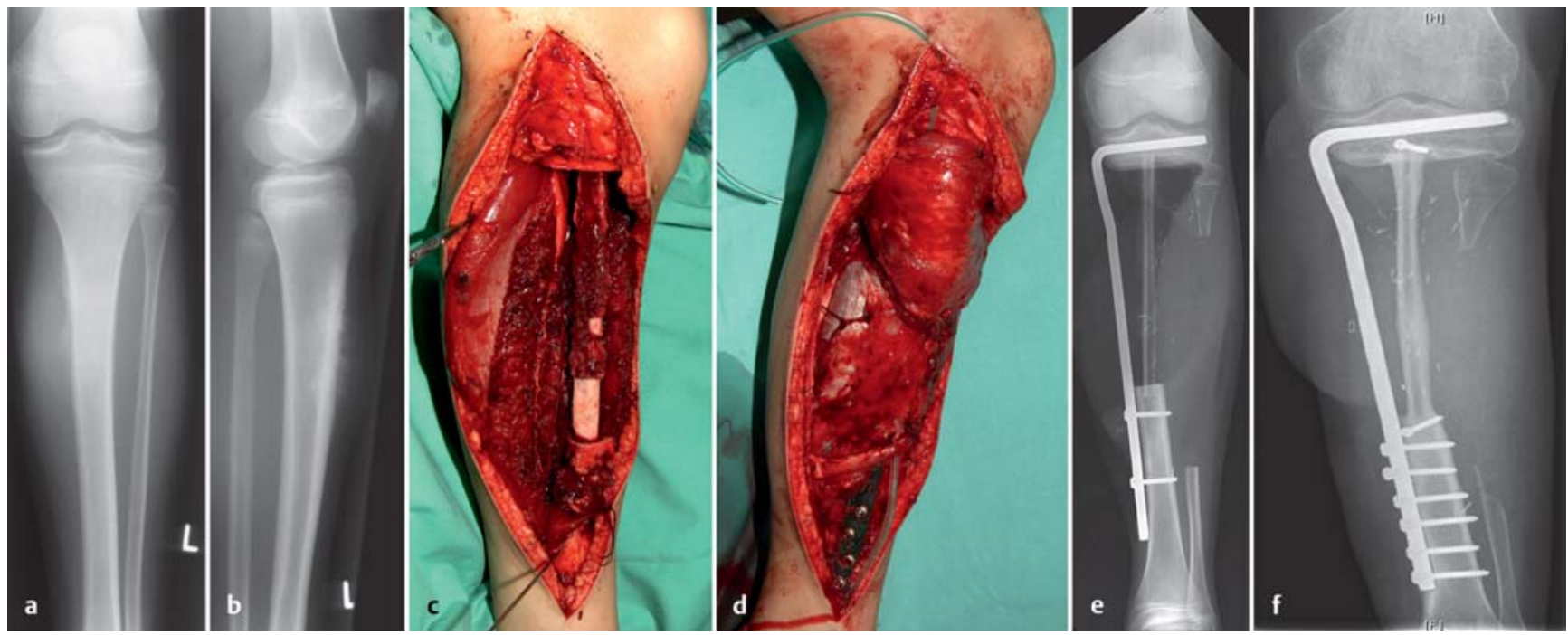

Abb. 2 a bis f a, b Osteosarkom proximale Tibia (a.-p. + lat.) mit den typischen radiologischen Zeichen: Codman-Dreiecke, lamellenartige Periostauflagerungen, Spiculae. c Fibula-pro-Tibiagraft nach Resektion der proximalen Tibia $(150 \mathrm{~mm})$ unter Erhalt der Epiphyse. d Plastische Rekonstruktion mit gefäßgestieltem Gastrocnemius-Muskellappen. e Postoperatives Röntgenbild nach Interpronation der ipsilateralen Fibula in die Tibia. $\mathbf{f}$ Im konventionellen Röntgenbild 2 Jahre postoperativ ist die beginnende Hypertrophie des Fibulainterpronats erkennbar.

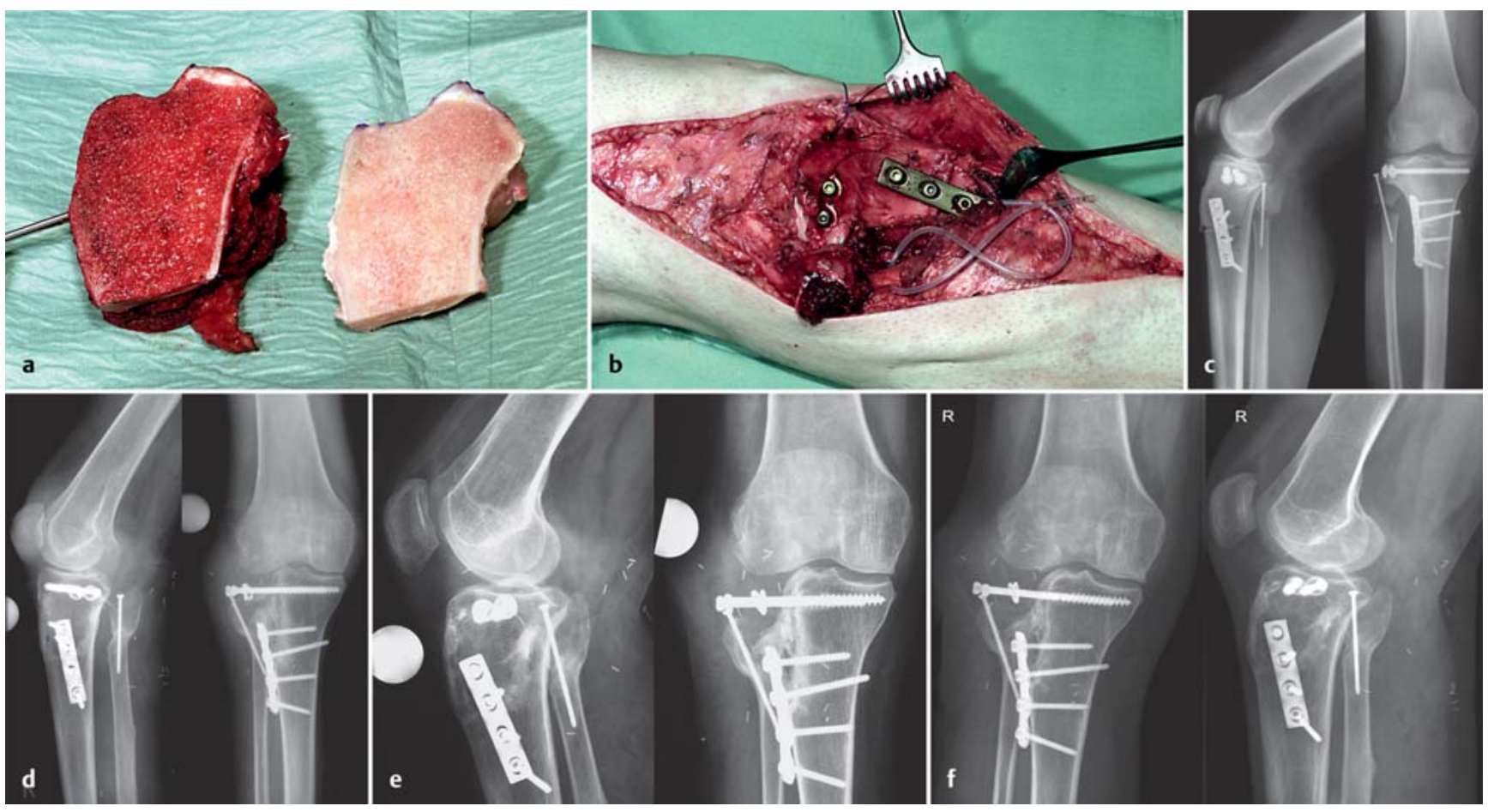

Abb. $\mathbf{3}$ a bis $\mathbf{f}$ a Anfertigung des formgenauen Allografts nach Resektion des Condylus lateralis tibiae bei Klarzellsarkom. b Implantation des Allografts am lateralen Tibiakondylus und Augmentation mittels Schrauben- und Plattenosteosynthese. c Postoperatives Röntgenbild nach Implantation des Allografts. d, e Zunehmende Resorption des tibialen Allografts bei verheilter Tibiaosteoteomie 2 + 3 Jahre postoperativ. f Neun Jahre postoperativ ist der Allograft nahezu komplett resorbiert.

In Abhängigkeit der Tumorentität und Lokalisierung ist eine intraläsionale, marginale, weite oder radikale Tumorresektion notwendig. Biologische Rekonstruktionen zeichnen sich durch Langlebigkeit durch Anpassung an veränderte mechanische Bealstungen und Längenwachstum aus.

\section{Hauptteil}

\section{Rekonstruktionsmöglichkeiten nach Resektion}

Die Wahl des Verfahrens muss bei jedem Patienten individuell getroffen werden. Prinzipiell sollten gerade beim Kind oder jungen Erwachsenen biologische Rekon- struktionen angestrebt werden. Entscheidend ist eine sorgfältige Selektionierung der Patienten. Bei Nebenerkrankungen wie Diabetes mellitus, Osteoporose, Hyperparathyreoidismus, Niereninsuffizienz, immunsuppressiv behandelten Patienten oder auch Nikotinabusus ist eine biologische Defektwiederherstellung häufig nicht erfolgreich durchzuführen. 
Von Anfang an muss der Patient in die Therapie integriert werden. Die ausführliche präoperative Aufklärung über den Eingriff und die notwendige postoperative Nachbehandlung, die in Abhängigkeit des gewählten Verfahrens mehrere Monate bis Jahre dauern kann, sollten dem Patienten und ggf. den Angehörigen in detaillierten Gesprächen erläutert werden. Letztendlich wird die Patientencompliance über den Erfolg oder Misserfolg einer biologischen Knochenrekonstruktion mitentscheiden.

\section{Autograft (Abb. 1)}

\section{Spongiosa}

Kleinere Defekte lassen sich durch autologe Spongiosa auffüllen. Hierzu eignen sich insbesondere intraläsionale Resektionen von benignen Tumoren. Wichtig ist, bei nicht ausgeschlossener Malignität eine Kontamination der Spenderregion mit dem Tumor zu vermeiden. Dies geht nur über separate Instrumente und Wechsel der OP-Kleidung oder aber über eine primäre Knochenentnahme und sekundäre Resektion. Tumorverschleppungen in den Bereich der Spongiosaentnahmestelle sind in der Literatur beschrieben.

\section{Gefäßgestielte Transplantate}

Die gefäßgestielte Fibula ist das am häufigsten verwendete Transplantat in der Tumorchirurgie. Im Bereich der Tibia kann die ipsilaterale Fibula in den Defekt hineingeschwenkt werden und so eine mikrochirurgische Gefäßanastomose vermieden werden. Die Stabilität der unteren Extremität ist bei Transplantation eines Fibulatransplantats erst nach Hypertrophie der Fibula gegeben. Dies kann je nach Alter des Patienten auch bis zu 24-36 Monate in Anspruch nehmen mit entsprechender Gefahr von Frakturen des Transplantats und/oder des Osteosynthesematerials. Junge Patienten zeigen ein deutlich schnelleres Hypertrophiepotenzial. Im Bereich der oberen Extremität ist ein Transplantat für die Stabilität in der Regel ausreichend. Um die Stabilität im Bereich der unteren Extremität zu erhöhen, besteht die Möglichkeit eines sog. Manteltransplantats. Hier wird um die eingebrachte Fibula ein kortikaler Allograft eingebracht. Die Erfahrungsberichte in der Literatur sind jedoch auf kleine Fallserien beschränkt. Alternativ kann eine 2. kontralaterale Fibula transplantiert werden. Die gefäßgestielte Fibula ist operationstechnisch anspruchsvoll und zeitaufwendig. Nachteilig bei bi-

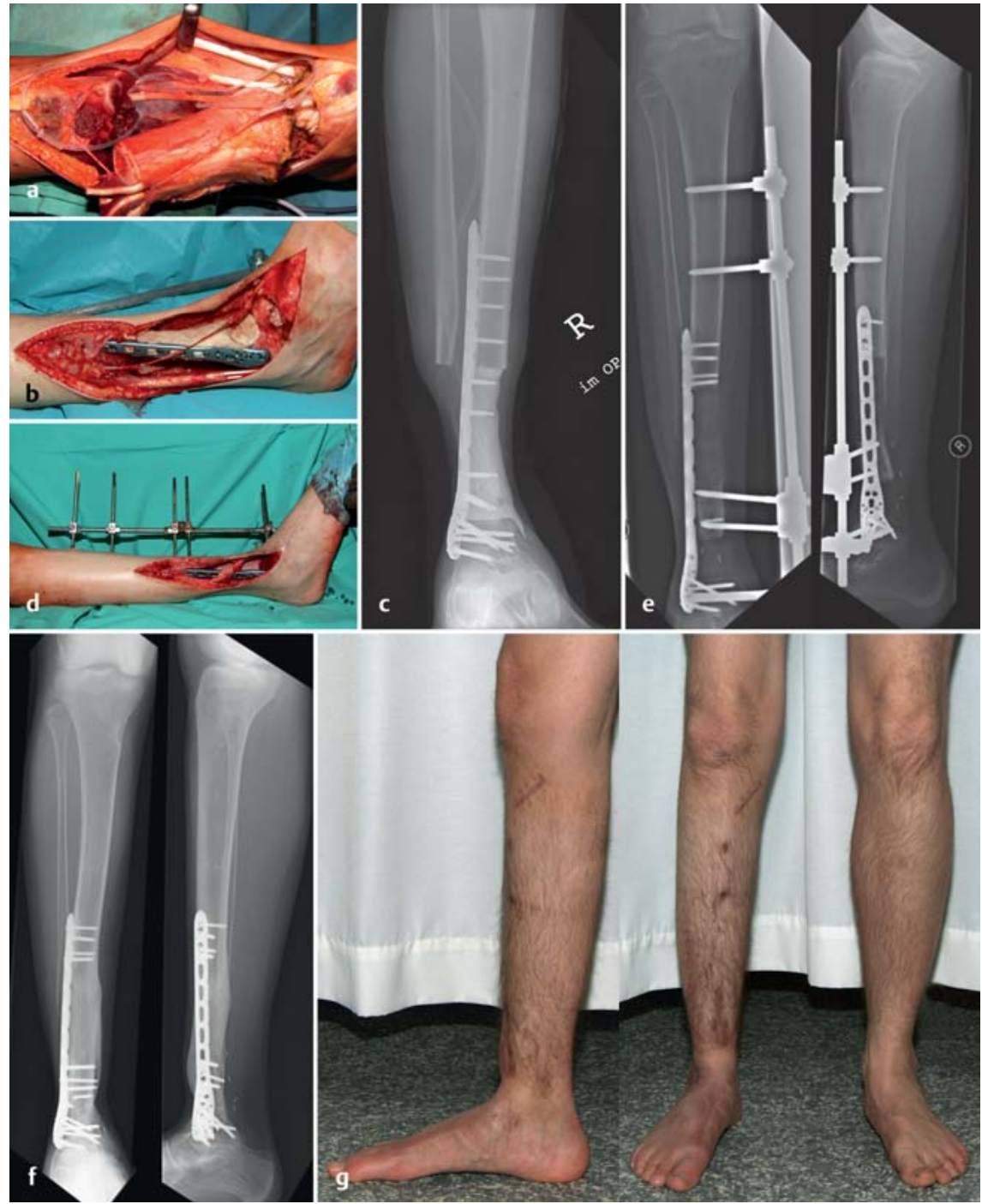

Abb. $\mathbf{4 a}$ bis $\mathbf{g}$ a En-bloc-Resektion der distalen Tibia und Fibula mit Resektion des anterioren Kompartiments und des M. flexor hallucis longus a. G. eines Osteosarkoms. b, c Implantation eines PMMA-Spacers über $10 \mathrm{~cm}$ als distaler Tibiaersatz und tibiotalare Stabilisation mittels winkelstabiler Platte. d Segmenttransport in Monorail-Technik mittels Regazzoni-Fixateur nach Explantation des PMMA-Spacers 10 Monate nach Tumorresektion und Abschluss der systemischen Chemotherapie. e Verlaufskontrolle des Segmenttransports mittels konventionellem Röntgenbild 60 Tage nach Transportbeginn. Klare Darstellung der Kalzifizierung des Regeneratknochens. f Der integrierte Regeneratknochen ist komplett tibiotalar integriert und füllt den ehemaligen Defekt komplett aus. g Vollbelastung 3 Monate nach Docking-OP und insgesamt 8 Monate nach Transportbeginn.

lateralem Transplantat ist der Eingriff an einer gesunden Extremität. Peronaeusschäden im Rahmen der Entnahme sind beschrieben. Eine Tumorverschleppung in gesunde Bereiche muss durch peinlichst genaue Vorsichtsmaßnahmen vermieden werden (s.o.) (Abb. 2).

Neben der freien Fibula eignen sich für kleinere Defekte auch gefäßgestielte Transplantate vom Beckenkamm oder der Skapula (hier ist die Kombination mit einem gefäßgestielten Muskellappen möglich). Am proximalen Humerus besteht bei sehr jungen Patienten die Möglichkeit der Clavicula-pro-humeri-Re- konstruktion. Die Klavikula wird hier vom sternalen Ende her mobilisiert und gestielt in den proximalen Humerus eingeschwenkt.

Die Transplantation von nicht gefäßgestielten kortikalen Transplantaten ist lediglich bis zu einer Länge von $5 \mathrm{~cm}$ empfohlen.

\section{Allograft}

Spongiöser Allograft eignet sich zur Auffüllung von metaphysären Defekten bei erhaltener Kortikalis. Bei speziellen Krankheitsbildern (z.B. fibröser Dyspla- 

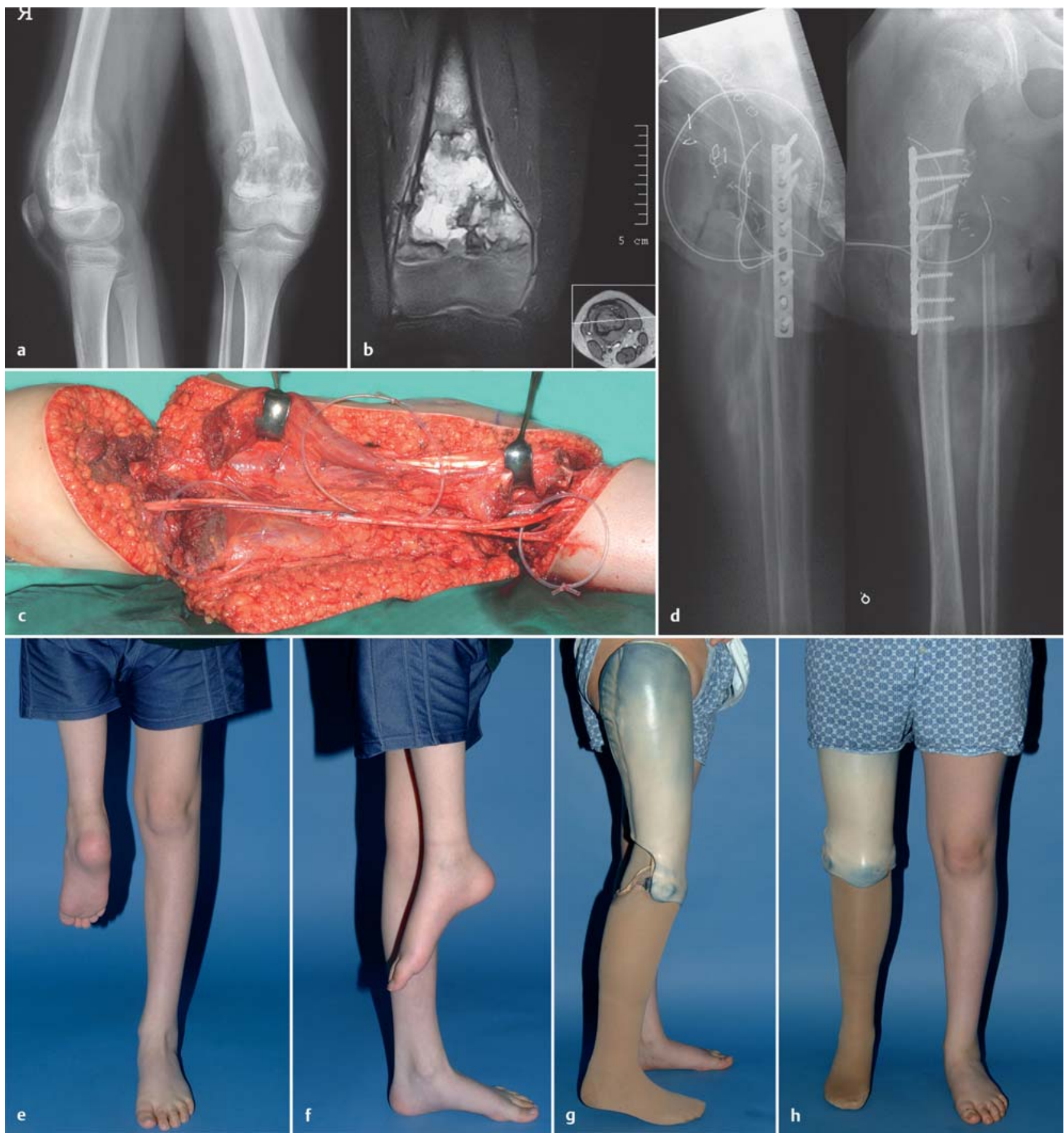

Abb. $\mathbf{5}$ a bis $\mathbf{h}$ a Darstellung des Osteosarkoms am distalen Femur rechts im konventionellen Röntgen. b Darstellung des Osteosarkoms am distalen Femur rechts im MRT. c Langstreckige Darstellung der A. femoralis/poplitea und des N. ischiadicus. d Postoperatives Röntgenbild: Nach Drehung des Unterschenkels um $180^{\circ}$ wurde die proximale Tibia an das proximale Femur mittels Plattenosteosynthese augmentiert. e, $\mathbf{f}$ Die Gelenkebene des oberen Sprunggelenks wurde exakt auf Höhe der kontralateralen Kniegelenksebene eingestellt. g, h Die individuell angepasste Prothese ermöglicht eine gute Funktion für Gang und Stand.

sie) bietet der allogene Knochen sogar Vorteile gegenüber dem Autograft. Bei Kindern zeigt der spongiöse Allograft eine recht gute Integration und umgeht das Problem der Spongiosaentnahme am wachsenden Skelett. Der kortikale Allograft zielt auf eine Wiederherstellung der ursprünglichen Anatomie hin.
Im Gelenkbereich kann der Allograft auch in Kombination mit einer gestielten Gelenkprothese eingesetzt werden oder als osteochondraler Allograft. Der autoklavierte Allograft besitzt zunächst keine lebenden Zellen und ist avaskulär. Findet eine Integration des kortikalen Allografts statt, so schreitet diese sehr schleichend voran. Nach 2 Jahren sind lediglich ca. $10 \%$ von Haver'schen Kanälen durchsetzt. Die Komplikationsrate bei kortikalen Allografts bez. Pseudarthrose, Infektion und Fraktur liegt teilweise über $30 \%$. Im deutschsprachigen Raum wird die Verwendung von kortikalen Allografts daher sehr restriktiv gehandhabt. 
Die beigefügte Bildserie präsentiert das Versagen eines Allografts am Condylus lateralis tibiae, der nach Resektion eines Klarzellsarkoms mittels Schrauben- und Plattenosteosynthese augmentiert worden war. Im zeitlichen Verlauf ist es zur Resorption des allogenen Knochentransplantats gekommen, eine ossäre Integration ist ausgeblieben (Abb. 3).

\section{Kallusdistraktion}

Die Defektfüllung durch Kallusdistraktion mittels Segmenttransport ist ein verbreitetes Mittel in der septischen und rekonstruktiven Chirurgie. Auch nach Tumorresektion ist eine Defektauffüllung durch Kallusdistraktion möglich. Um bei notwendiger Chemotherapie das Infektrisiko durch den externen Transport zu verringern, besteht die Möglichkeit der sekundären Rekonstruktion. Initial ist eine Stabilisierung mit temporärer Zementspacer-Implantation sinnvoll. Zum einen wird hierdurch die Osteosynthese zusätzlich augmentiert, zum anderen wird der Membran um den Knochenzement herum osteogene Aktivität zugesprochen. Nach Abschluss der Chemotherapie kann unter sichereren Bedingungen mit der Rekonstruktion begonnen werden. Nachteilig bei diesem Verfahren ist eine über Monate nicht belastungsstabile Extremität. Der große Vorteil besteht in der biologischen Rekonstruktion ohne Entnahmemorbidität. Technische Erfahrungen in der Extremitätenrekonstruktion sind für den Operateur zwingend erforderlich (Abb.4).

\section{Extrakorporale Verfahren}

Die extrakorporale Hochdosisstrahlentherapie ist eine Methode, bei der der resezierte Tumor extrakorporal einer Hochdosisradiatio zugeführt und anschließend wieder implantiert wird. Es wird durch die Bestrahlung von einer kompletten Zerstörung aller Zelllinien und hier insbesondere der neoplastischen Zellen ausgegangen. Die knöcherne Matrix soll ähnlich dem Allograft ein Gerüst für das Einwachsen neuer Stammzellen und deren Differenzierung zu Osteoblasten bieten. Nachteilig ist wie beim Allograft das hohe Risiko einer Infektion, Fraktur und Pseudarthrose. Eine histopathologische Aufarbeitung der Resektionsgrenzen ist leider nicht gegeben. Langzeitergebnisse zu dieser Methode fehlen. Eine ähnliche Methode ist die extrakorporale Kryotherapie mit flüssigem Stickstoff. Auch diese ist jedoch bisher keiner breiten Evaluation unterzogen.

\section{Alternative Extremitätenrekonstruktion}

Erstmals 1930 führte Borggreve die Umkehrplastik durch, die im Jahr 1981 durch Salzer in die Tumorchirurgie übernommen wurde. Winkelmann modifizierte die Umkehrplastik in den 80erJahren, sodass Tumoren des gesamten Femurs, der proximalen bis mittleren Tibia und des unteren Beckenbereichs addressiert werden konnten und eine primäre Amputation vermieden werden konnte. Prinzipiell wird bei der Umkehrplastik ein Segment des Femurs oder der proximalen Tibia reseziert, der Unterschenkel bzw. das obere Sprunggelenk um $180^{\circ}$ gedreht und auf Höhe des Kniegelenks mittels femorotibialer Osteosynthese fixiert. Kontraindikationen für die Umkehrplastik sind die Tumorinfiltration des N. ischiadicus und eine periphere arterielle Verschlusskrankheit (Abb. 5).

Seit Einführung der modularen Tumorendoprothesen ist die Umkehrplastik eine seltenere Operation geworden, die aber in bestimmten Konstellationen ihre uneingeschränkte Indikation findet. So kann aufgrund der Tumorausdehnung und -lokalisation in bestimmten Fällen eine sinnvolle weite onkologische Resektion nicht extremitätenerhaltend durchgeführt werden. Bei Kindern stellt die Umkehrplastik eine gute Alternative zur Implantation einer Wachstumsprothese dar, die zahlreiche Folgeoperationen zur Prothesenverlängerung (9-15 Operationen) notwendig macht. Während es sich bei der Umkehrplastik um einen - abgesehen von einer potenziellen Implantatentfernung - einmaligen Eingriff handelt, stellen die zahlreichen Reoperationen ein großes Infektionsrisiko bei allen Tumorprothesen dar. Eine weitere Indikation, auch beim Erwachsenen, ist der fehlgeschlagene Extremitätenerhalt, insbesondere bei chronischen Infektionen und/oder ausgedehntem Verlust an Knochensubstanz bzw. unzureichendem Weichteilmantel. Nach individueller Anpassung einer Prothese, die mit dem Sprunggelenk als Neokniegelenk bewegt werden kann, sind hervorragende funktionelle Ergebnisse zu erzielen.

Bereits 1950 veröffentliche Van Nes erstmals Langzeitergebnisse, die dem Verfahren ein gutes funktionelles Ergebnis bescheinigen, denn die Patienten erreichen ein hohes Aktivitätsniveau in Alltag, Sport- und Berufsfähigkeit. An die kosmetische Veränderung gewöhnen sich die Patienten relativ schnell, sodass sie ein weitgehend normales Le- ben ohne psychosoziale Störungen führen können.

\section{Verzicht auf Knochenrekonstruktion}

In einigen Fällen kann auf eine knöcherne Rekonstruktion nach Knochentumorresektion verzichtet werden und die Modifikation der Belastung der Extremität oder eine Immobilisation kann ausreichend sein. Hierbei wird erneut auf die Mitarbeit des Patienten verwiesen. Temporär geschwächter Knochen kann mit einer zeitweise stabilisierenden Osteosynthese versorgt werden.

\section{Schlussfolgerung}

Die Entscheidung, welches rekonstruktive Verfahren nach Knochentumorresektionnen zu wählen ist, muss patientenindividuell getroffen werden und ist von zahlreichen Faktoren abhängig. Die technisch anspruchsvollen Eingriffe bleiben dem erfahrenen Chirurgen vorbehalten. Die Patienten sind noch vor dem operativen Eingriff über die notwendige Nachbehandlung zu informieren und eine frühzeitige Integration in die Therapie ist absolut notwendig. Biologische Rekonstruktionen zeichnen sich durch ihre Langlebigkeit, prothetische Lösungen durch ihre Primärstabilität aus.

\section{Literatur}

${ }^{1}$ Freyschmidt J, Ostertag H, Jundt G. Textbook of Bone Tumors. Berlin, Heidelberg: Springer; 2010

2 Hardes J, Gebert C, Hillmann A et al. [Rotationplasty in the surgical treatment plan of primary malignant bone tumors. Possibilities and limits]. Orthopade 2003; 32: 965-970

3 Simon MA, Springfield DS. Surgery for Bone and Soft-tissue Tumors. Philadelphia, PA: Lippincott-Raven; 1998

4 Raskin KA, Hornicek F. Allograft reconstruction in malignant bone tumors: indications and limits. Recent Results Cancer Res 2009; 179: $51-58$

${ }^{5}$ Kunz P, Bernd L. Methods of biological reconstruction for bone sarcoma: indications and limits. Recent Results Cancer Res 2009; 179: 113-140

\section{Ulf Brunnemer \\ Assistenzarzt \\ Prof. Dr. med. Christian Krettek \\ Direktor der Klinik \\ Prof. Dr. med. Thomas Gösling \\ Ltd. Oberarzt der Klinik}

Unfallchirurgische Klinik

Medizinische Hochschule Hannover

Carl-Neuberg-Straße 1

30625 Hannover

brunnemer.ulf@mh-hannover.de 